\title{
A pedunculated mass of the thigh
}

A 61-year-old Chinese woman presented to the general surgery clinic for a long-standing lump over her proximal medial right thigh. The painless lump was first noticed 15 years ago and had been slowly enlarging since. She sought medical attention due to increasing discomfort while sitting and walking, as well as difficulty getting dressed. Clinical examination revealed a large pedunculated mass at the proximal medial right thigh measuring $15 \times 10 \mathrm{~cm}$ (Fig. 1). It was soft, non-tender, and freely mobile in the subcutaneous plane. The mass did not show overlying skin discoloration or ulceration, nor was it transilluminable. A magnetic resonance imaging (MRI) study was arranged to further characterise the mass and delineate its local extent. The MRI showed a pedunculated subcutaneous mass that was predominantly hyperintense on T1-weighted sequence and hypointense on fat-saturated sequence (Fig. 2). The tumour contained several enhancing septa (Fig. 2), as well as a small focus of susceptibility indicating haemorrhage or calcification. There was no invasion of the underlying muscle fascia.

What is the likely diagnosis?
A. Dermatofibrosarcoma protuberans
B. Neurofibroma
C. Myxoid liposarcoma
D. Fibrolipoma
E. Skin tag (acrochordon)

The patient subsequently underwent an excisional biopsy of the mass whereupon an encapsulated fatty tumour was encountered. The tumour, together with its stalk, was resected en bloc (Fig. 3). Histological examination confirmed a lipomatous tumour composed of lobules of mature fat separated by fibrous bands. The specimen tested negative for murine double minute 2 (MDM2) amplification, which was consistent with a benign lipomatous tumour. A final diagnosis of fibrolipoma was made.

Answers A to E are relevant differentials for a superficial lump that can be further narrowed down by thorough clinical assessment and appropriate imaging. Acrochordon-also known as skin tag, cutaneous papilloma or fibroepithelial polyp - arises from the dermis and may be skin-coloured or hyperpigmented. It is a common benign lesion that is usually treated for

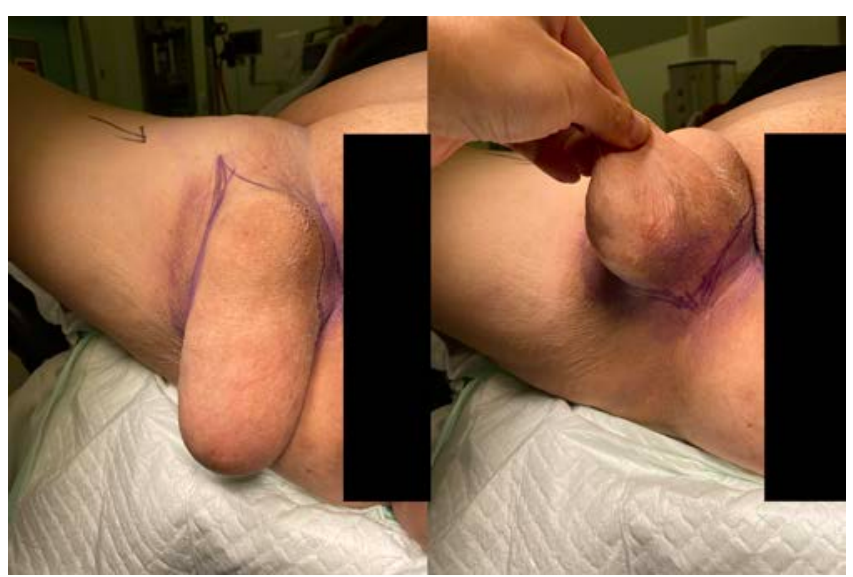

Fig. 1. Large pedunculated mass without skin changes or fixation.

cosmesis. Dermatofibrosarcoma protuberans (DFSP) is a low-grade sarcoma arising from the dermissubdermis, most commonly found on the trunk. It presents as a slow-growing skin nodule or plaque that may ulcerate when large. On MRI, DFSP is isointense to muscle on T1-weighted (T1W) sequence, hyperintense on T2-weighted (T2W) sequence and shows variable enhancement. Wide-excision is the mainstay of treatment for DFSP. Neurofibroma is a benign peripheral nerve sheath tumour that may occur sporadically (solitary) or as part of neurofibromatosis type 1, an autosomal dominant neurocutaneous syndrome. Cutaneous neurofibromas are firm, skin-coloured nodules that may be painful and cause symptoms related to masseffect. The characteristic button-hole sign describes the invagination of a cutaneous neurofibroma on compression by the fingertip. On MRI, neurofibromas are circumscribed enhancing lesions that may be seen in continuity with the involved nerve. They are hyperintense on T2W sequence and can show a "target" appearance consisting of central hypointensity and peripheral hyperintensity. When large $(>5 \mathrm{~cm})$ or newly symptomatic, the possibility of a malignant peripheral nerve sheath tumour (MPNST) should be considered. MPNSTs can occur de novo or from de-differentiation of a benign tumour. Myxoid liposarcoma is the second most common liposarcoma subtype after well-differentiated liposarcoma. Lesions usually arise in the deep muscular or fascial spaces of the lower limb. In contrast to most lipomatous lesions, myxoid liposarcomas are predominantly hypointense on T1W 


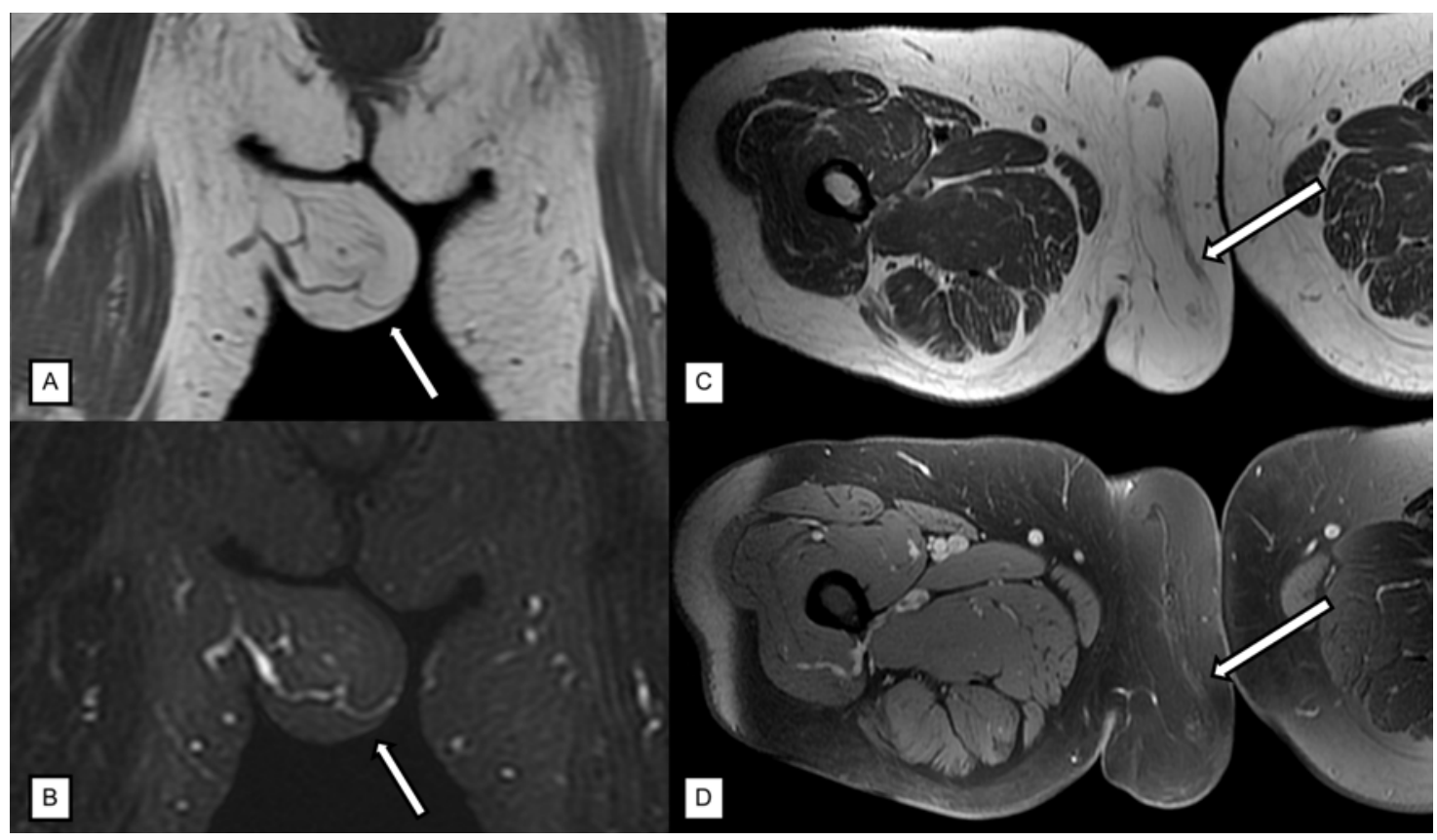

Fig. 2. (A) Coronal T1-weighted image shows a hyperintense pedunculated subcutaneous mass (arrow). (B) Coronal short tau inversion recovery image shows corresponding signal suppression, indicating a predominantly fatty composition. (C) Axial T1-weighted image shows a thick septum (arrow) that faintly enhances on (D) axial post-contrast fat-saturated sequence.

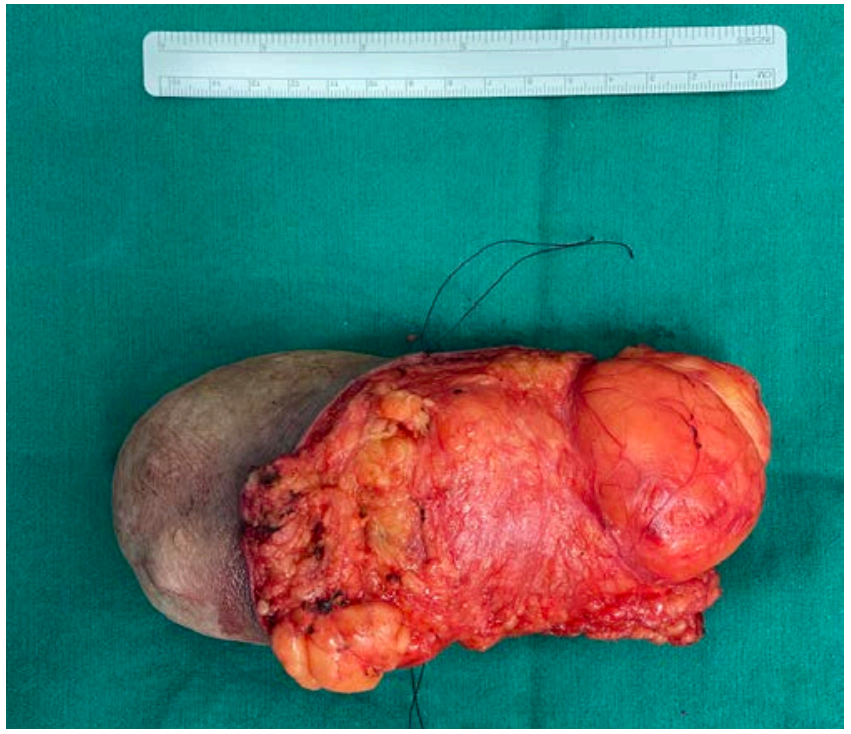

Fig. 3. Surgical specimen shows fatty composition of the tumour.

sequence and hyperintense on $\mathrm{T} 2 \mathrm{~W}$ sequence owing to a myxoid matrix. Small T1W hyperintense foci of fat may be seen and lesions may show homogeneous or heterogeneous enhancement.

Discussion. Lipomas are benign tumours of mesenchymal origin composed of mature adipose tissue. They are exceedingly common and account for up to
$48.1 \%$ of benign soft tissue tumours. ${ }^{1}$ Various benign histologic variants exist, including angiolipoma, fibrolipoma, myxolipoma, chondroid lipoma and myelolipoma. ${ }^{2}$ While generally small and asymptomatic, lipomas can sometimes be painful or result in compressive symptoms, thus prompting surgical intervention. Deep intramuscular or intrabdominal lesions often go unnoticed, presenting only when they have attained a massive size. Giant lipomas are so termed when they exceed $10 \mathrm{~cm}$ in size (as in the current case) or $1,000 \mathrm{~g}$ in weight. ${ }^{3}$

Superficial lipomas are usually diagnosed clinically. If in doubt, ultrasound is an accessible and inexpensive tool to confirm the diagnosis. On ultrasound, lipomas appear as circumscribed iso-hyperechoic lesions with little to no vascularity. Nonetheless, MRI should be performed for all large $(>5 \mathrm{~cm})$ or deep lesions. ${ }^{4}$ This permits assessment for atypical features as well as surgical planning. Whereas a lipoma appears as a welldefined lesion exhibiting MRI signal characteristics that mirror normal adipose tissue, a liposarcoma may show complex enhancing septa, prominent nonfatty soft tissue components and frank invasion. Atypical lipomatous tumour (ALT)/well-differentiated liposarcoma (WDL) is a low-grade malignancy that shows intermediate imaging features such as thick septa or patchy non-fatty components. Lesions in the 
extremities are termed ALTs, while those in the deeper spaces like the retroperitoneum are termed WDLs. Though histologically identical, clinical outcomes between ALT and WDL are vastly different because of the difficulty obtaining adequate resection margins for deep-seated WDLs. ${ }^{4}$ In practice, the imaging appearances of lipoma and ALT frequently overlap, and histological evaluation must be pursued where there is concern for ALT. To this end, molecular testing for MDM2 gene amplification has become a standard adjunctive tool for the distinction between lipoma and ALT. MDM2 amplification is consistently detected in ALT, but should not be present in lipoma.

Fibrolipoma is a rare benign variant characterised histologically by mature adipose lobules interspersed by broad fibrous bands. ${ }^{7}$ MRI cannot reliably differentiate fibrolipoma from ALT as both entities can be expected to show thick septa - a finding that should prompt histological evaluation. Interestingly, pedunculated fibrolipomas appear to have a predilection for the buttock and thigh. ${ }^{8-10}$ Clinical and imaging specialists alike should be alert to the possibility of a fibrolipoma when a pedunculated mass is encountered in this region.

\section{REFERENCES}

1. Myhre-Jensen O. A consecutive 7-year series of 1331 benign soft tissue tumours. Clinicopathologic data. Comparison with sarcomas. Acta Orthop Scand 1981;52:287-93.

2. Weiss SW. Lipomatous tumors. Monogr Pathol 1996;38:207-39.
3. Sanchez MR, Golomb FM, Moy JA, et al. Giant lipoma: case report and review of the literature. J Am Acad Dermatol 1993; 28(2 Pt 1):266-8.

4. Johnson CN, Ha AS, Chen E, et al. Lipomatous Soft-tissue Tumors. J Am Acad Orthop Surg 2018;26:779-88.

5. Brisson M, Kashima T, Delaney D, et al. MRI characteristics of lipoma and atypical lipomatous tumor/well-differentiated liposarcoma: retrospective comparison with histology and MDM2 gene amplification. Skeletal Radiol 2013;42:635-47.

6. O'Donnell PW, Griffin AM, Eward WC, et al. Can Experienced Observers Differentiate between Lipoma and Well-Differentiated Liposarcoma Using Only MRI? Sarcoma 2013;2013:982784.

7. Shin SJ. Subcutaneous fibrolipoma on the back. K Craniofac Surg 2013;24:1051-3.

8. Nogita T, Wong TY, Hidano A, et al. Pedunculated lipofibroma: A clinicopathologic study of thirty-two cases supporting a simplified nomenclature. J Am Acad Dermatol 1994;31(2 Pt 1):235-40.

9. Simsek T, Sonmez A, Aydogdu IO, et al. Giant fibrolipoma with osseous metaplasia on the thigh. J Plast Reconstr Aesthet Surg 2011;64:e125-7.

10. Xue EY, Schultz JJ, Datiashvili R. A Pedunculated Neoplasm of the Thigh. Eplasty 2017;17:ic20.

Chern Yue Glen Ong ${ }^{1}$ FRCR, Rui-Ming Teo ${ }^{2}$ MRCS, Yonghan Ting ${ }^{1}$ FRCR

${ }^{1}$ Department of Diagnostic Radiology, Tan Tock Seng Hospital, Singapore

${ }^{2}$ Department of General Surgery, Tan Tock Seng Hospital, Singapore

Correspondence: Dr Chern Yue Glen Ong, Department of Diagnostic Radiology, Tan Tock Seng Hospital, 11 Jalan Tan Tock Seng, Singapore 308433.

Email: glen_ong@ttsh.com.sg 